\title{
Modeling of a glass mineral wool process in view of Life Cycle Analysis
}

S. GERBINETa , V. BRIARD ${ }^{\mathrm{b}}$, and A. LÉONARDa

saicha.gerbinet@ulg.ac.be

aUniversity of Liège

LABORATORY of CHEMICAL ENGINEERING

Processes and Sustainable development

${ }^{b}$ Knauf Insulation 


\section{Contents}

1. Introduction

2. The production process

3. LCA and modeling

4. Results and advantages

5. Conclusion 


\section{Contents}

1. Introduction

2. The production process

3. LCA and modeling

4. Results and advantages

5. Conclusion 


\section{Knauf Insulation}

MINERAL WOOL

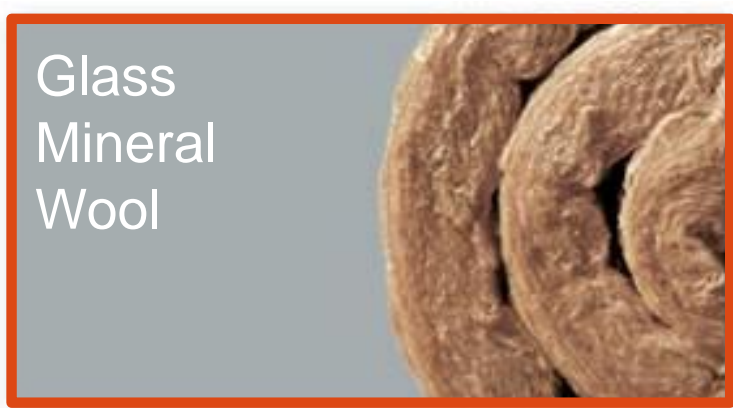

with ECOSE

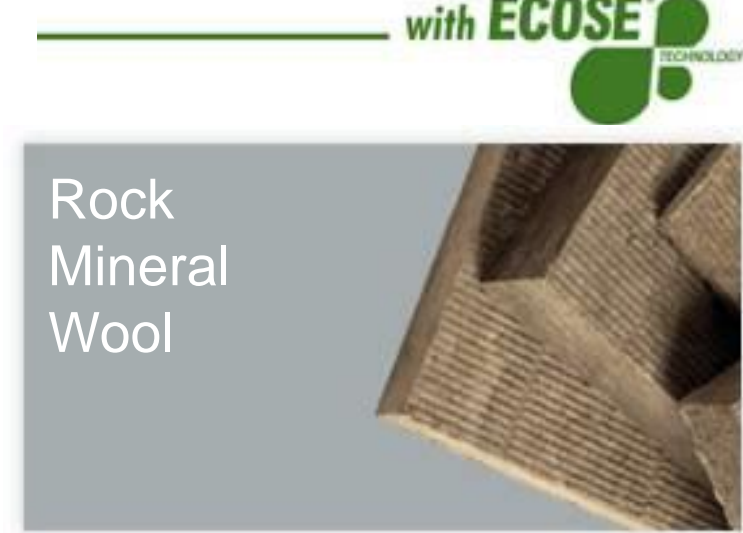

PLASTIC FOAMS

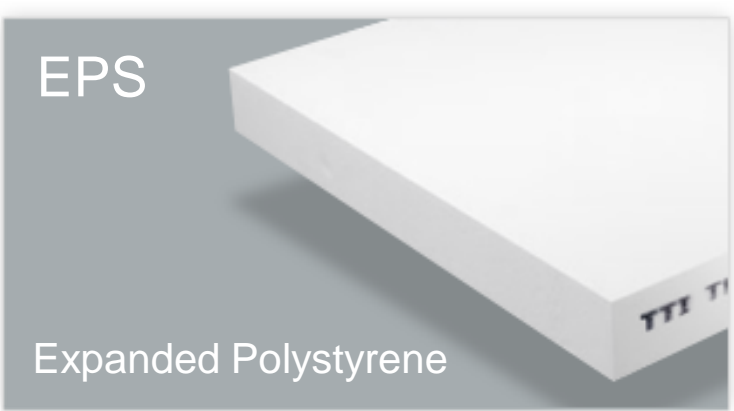

\section{XPS}

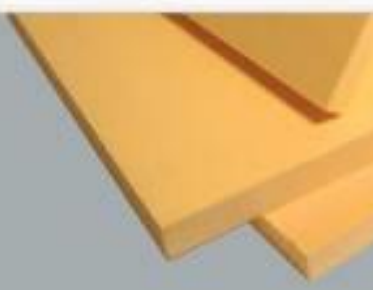

Extruded Polystyrene

\section{WOOD WOOL}
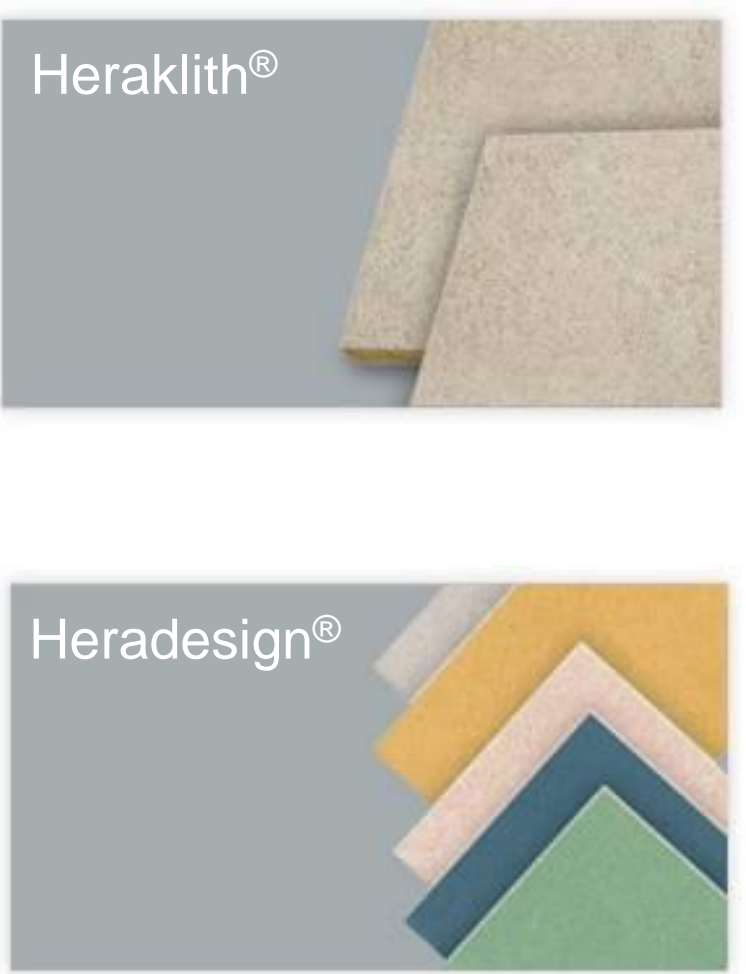

\section{5




\section{Knauf Insulation}

- LCA to Knauf Insulation

- First: market demand (Environmental Product Declaration (E.P.D.), etc.) 


\section{KNAUF|WEULATION}

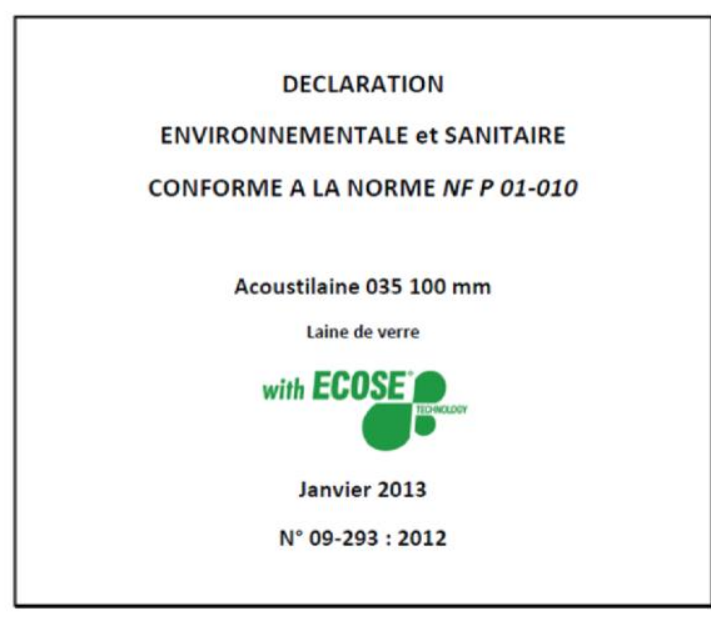

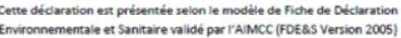

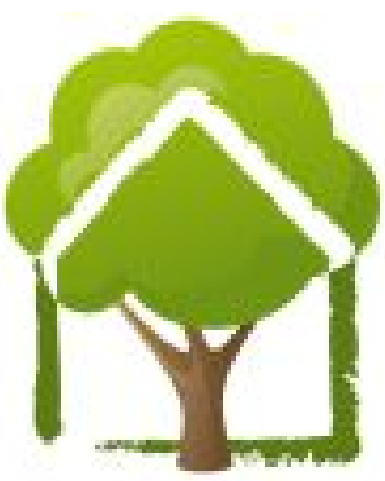

Institut Bauen und Umwelt e.V.

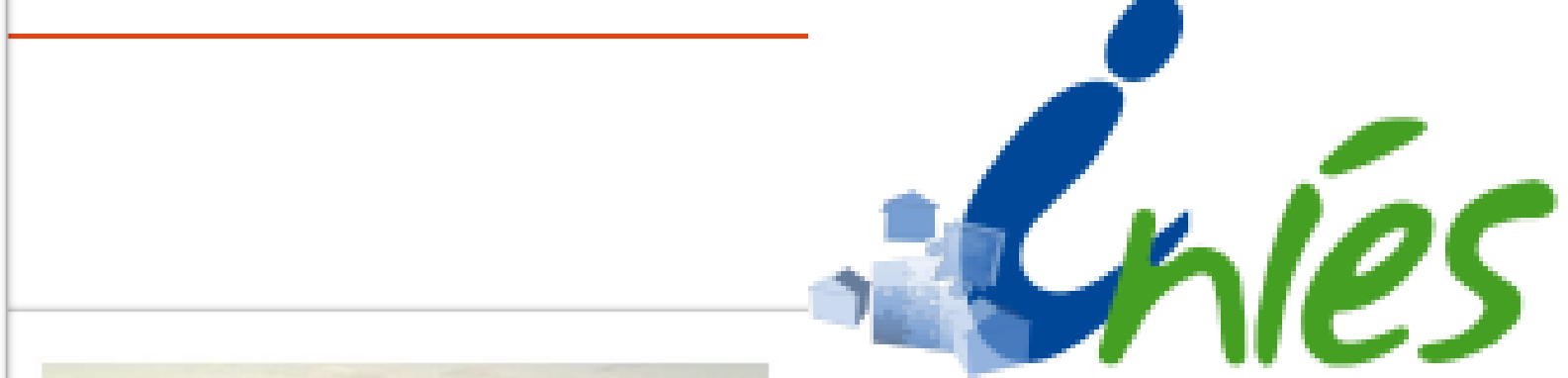

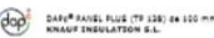

DAPC ${ }^{\circledR 0} 01.006$ PANEL PLUS (TP 138)
Umwelt-Produktdeklaration

nach is 014025

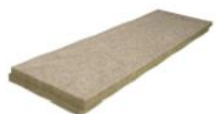

Holzwolle-Mehrschichtplatten mit Steinwollekern

Heraklith.

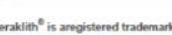

kиäpusum

Deklarationsnummer
EPD-KN1-2011711-D

Institut Baven und Umwelt e.

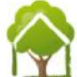

nuww. bav-umwett com

\section{de $100 \mathrm{~mm}$}

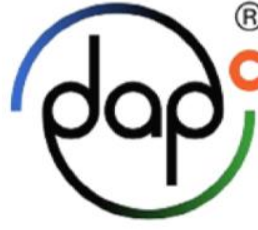

DE ACUERDO CON LAS NORMAS B) soness

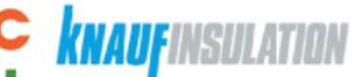

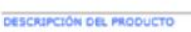
Panel seminigido de Lana Mineral de Vidrio

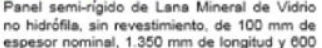
mm de anchura

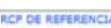
RCP001 - Productos aislantes tér

RUNTA Producciom KNAUF INSULATION LANNEMEZAN SAS Fr-65300 Lannemezan (France)

$\begin{array}{ll}\text { vaudotz } & \\ \text { Desde: } \quad 31.01 .2013\end{array}$ $\begin{array}{ll}\text { Desde: } & \begin{array}{l}31.01 .2013 \\ \text { Hasta: }\end{array} \quad 30.01 .2018\end{array}$

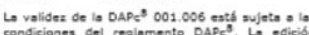

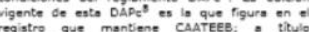

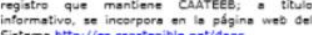




\section{Knauf Insulation}

- LCA to Knauf Insulation

- First: market demand (Environmental Product Declaration (E.P.D.), etc.)

a Then ECO-DESIGN 


\section{Contents}

1. Introduction

2. The production process

3. LCA and modeling

4. Results and advantages

5. Conclusion 


\section{The production process}

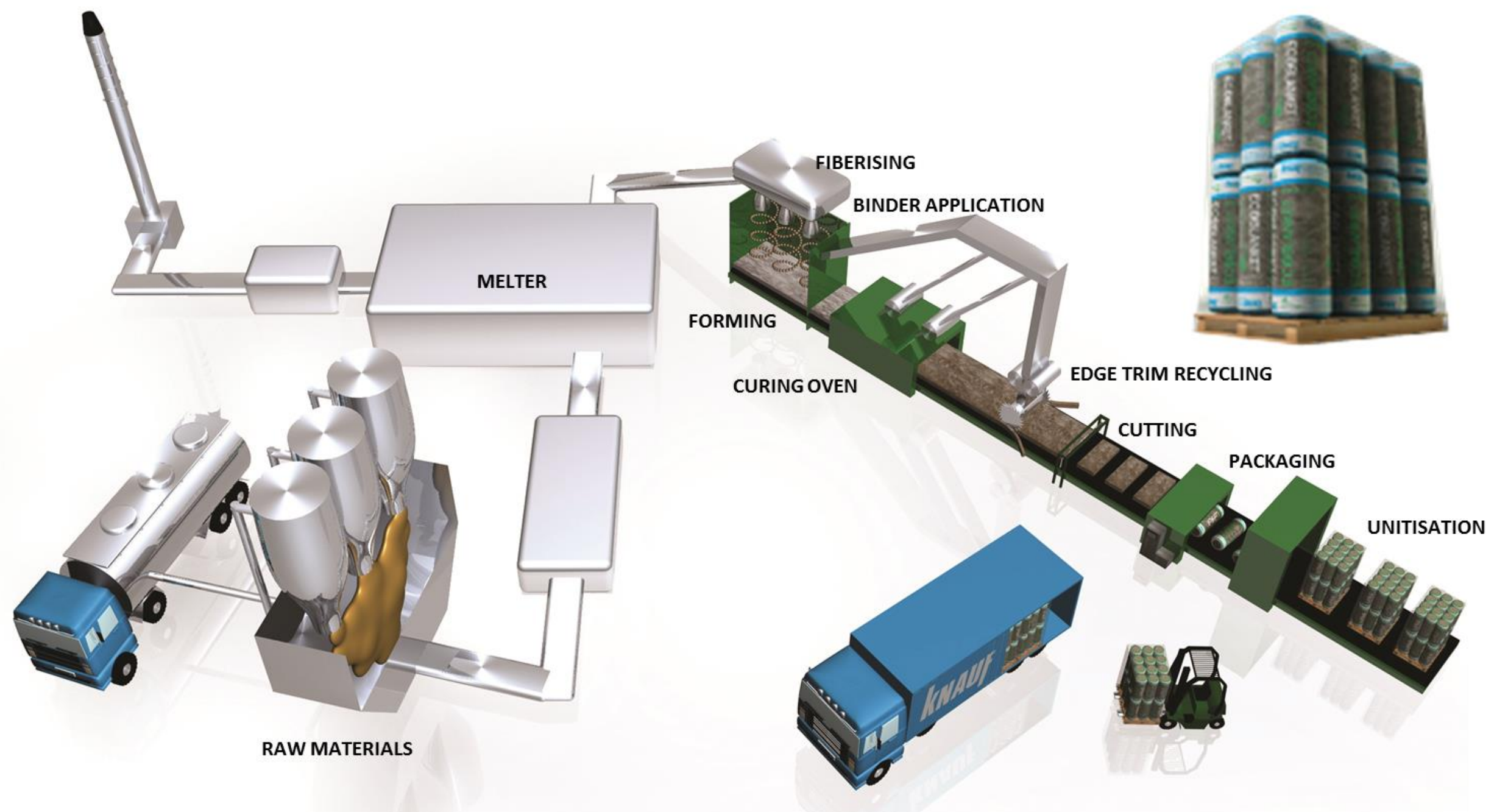

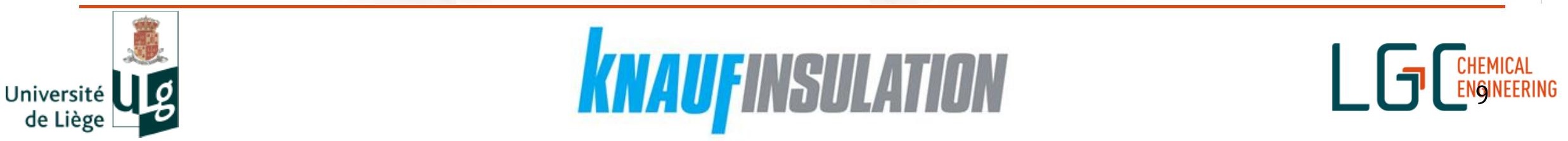




\section{The production process}

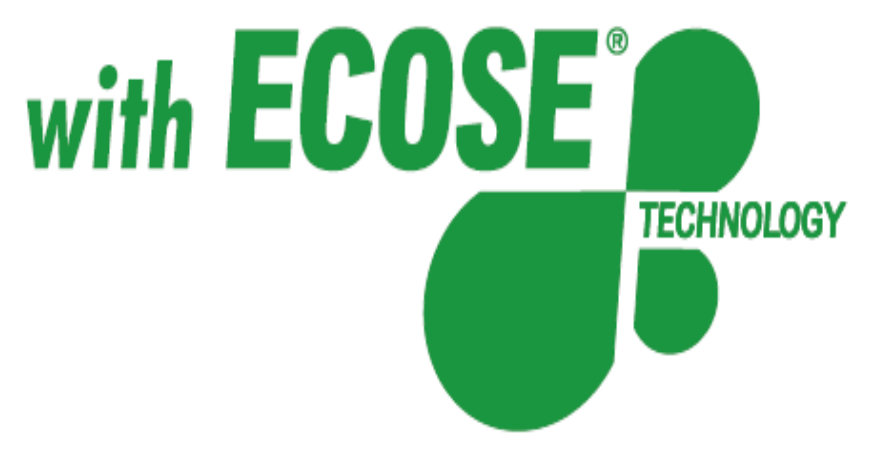

Binder : formaldehyde $\rightarrow$ bio-based from plant starch 


\section{Contents}

1. Introduction

2. The production process

3. LCA and modeling

4. Results

5. Advantages

6. Conclusion 


\section{LCA methodology}

- Special attention to allocation procedure (economic)

- Functional unit: production of $1 \mathrm{~m}^{3}$ of a specific product

- Use phase not included 


\section{Modeling}

- In GaBi 6

a Plans of different levels

- In a plan: other plans or processes.

- Flow to link plans and/or processes

- General operation principle: identical in all plants

$\rightarrow$ A unique model

$\rightarrow$ Structured // plant organization 


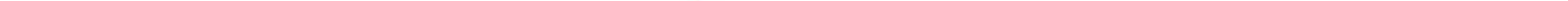




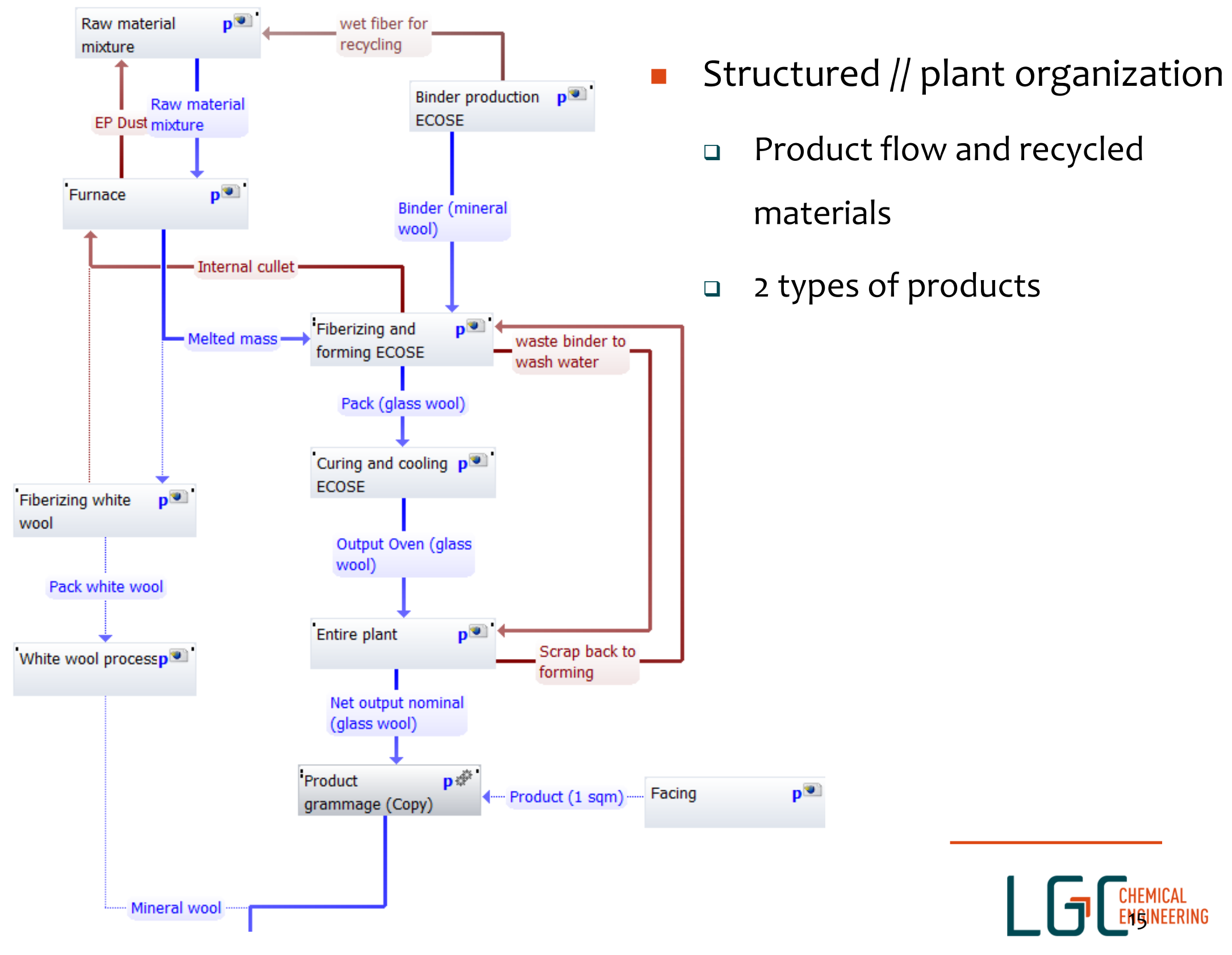




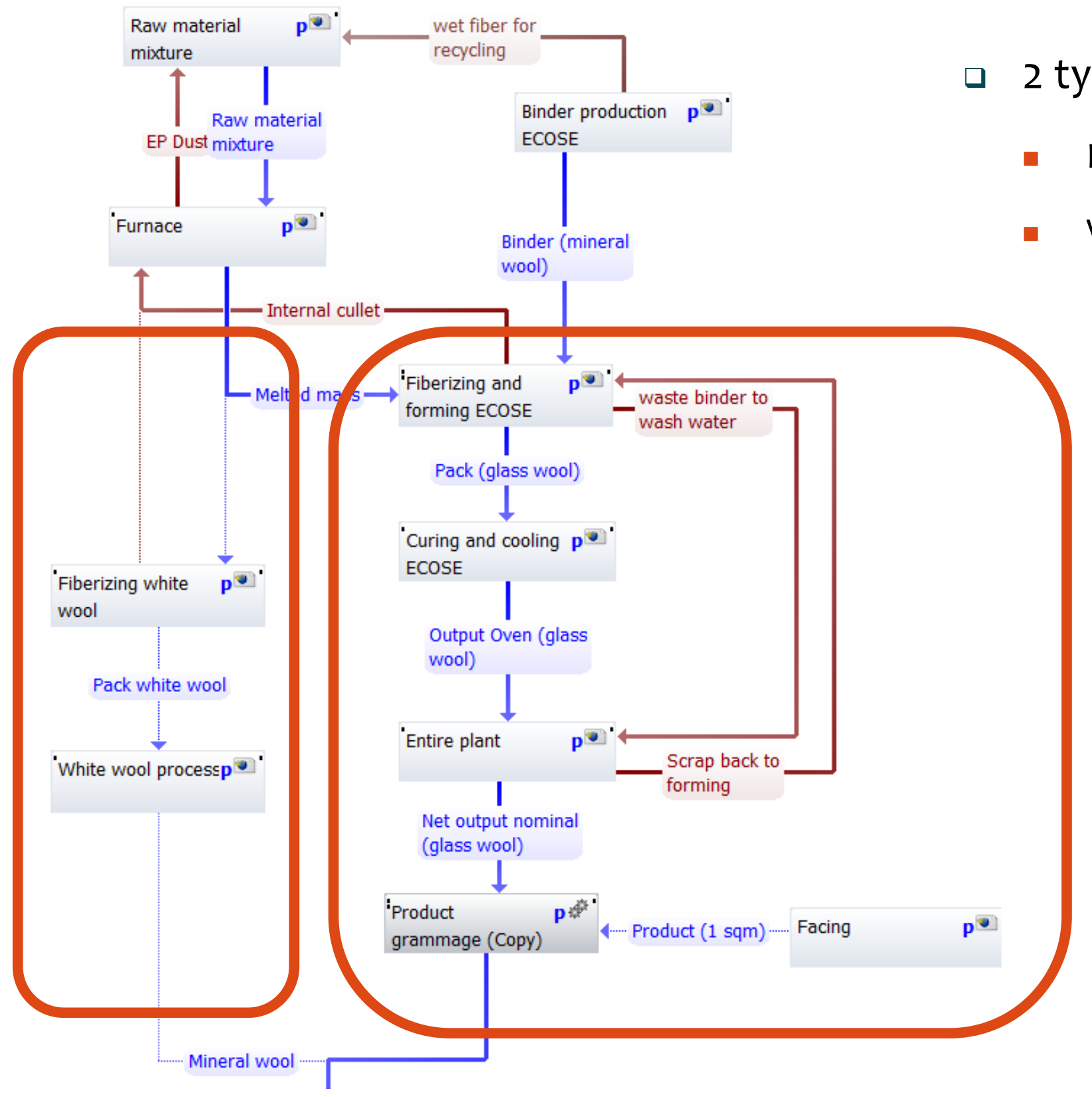

- Blowing wool

- Wool 


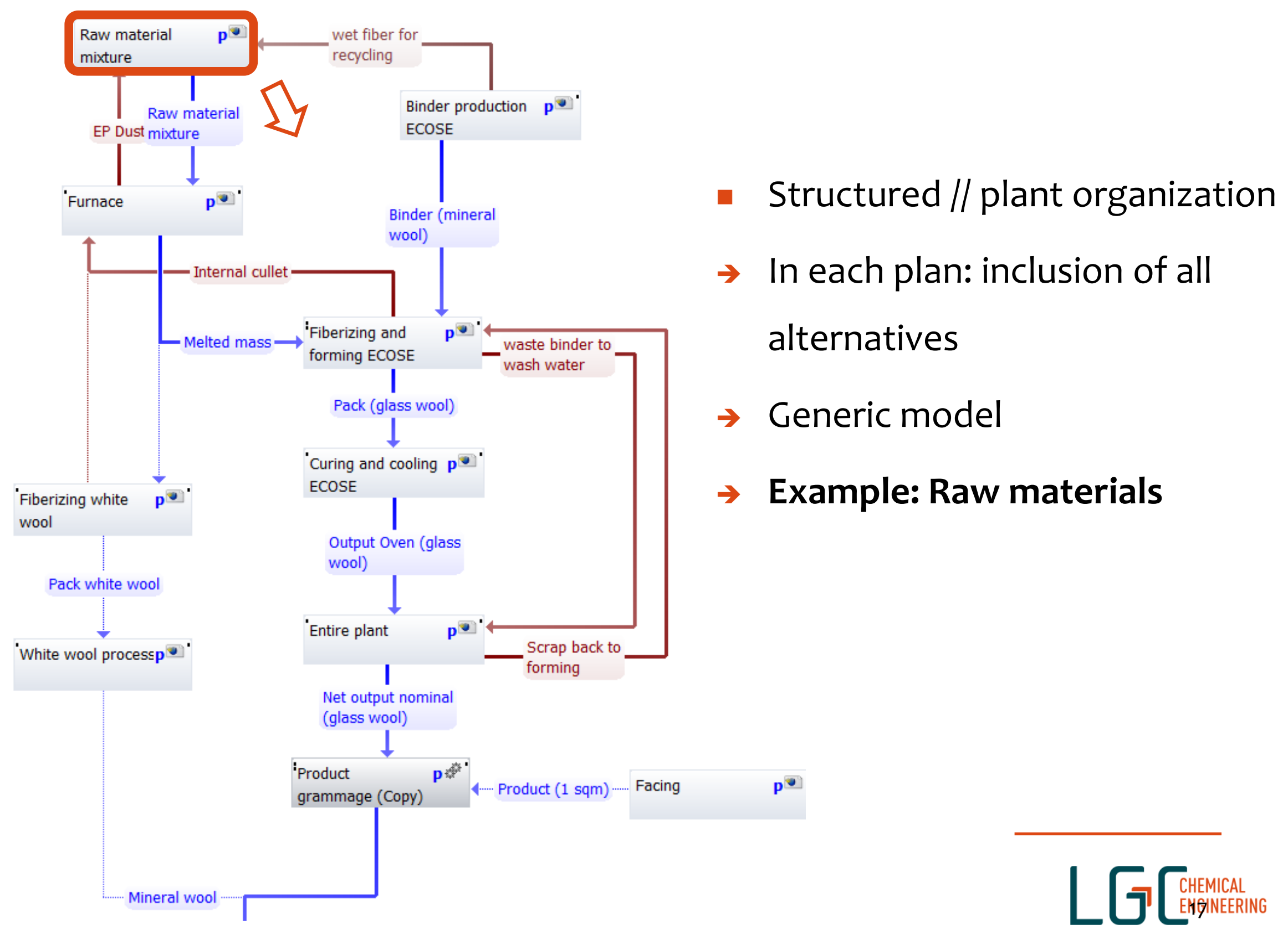




\section{Raw material mixture plan}

- Includes all possible (potential) raw materials

- Electric Energy Mixer: allows to choose the electricity grid mix

${ }_{R}$ - In the process parts where other energy sources are used (natural gas and/or oxygen), the same principle is applied

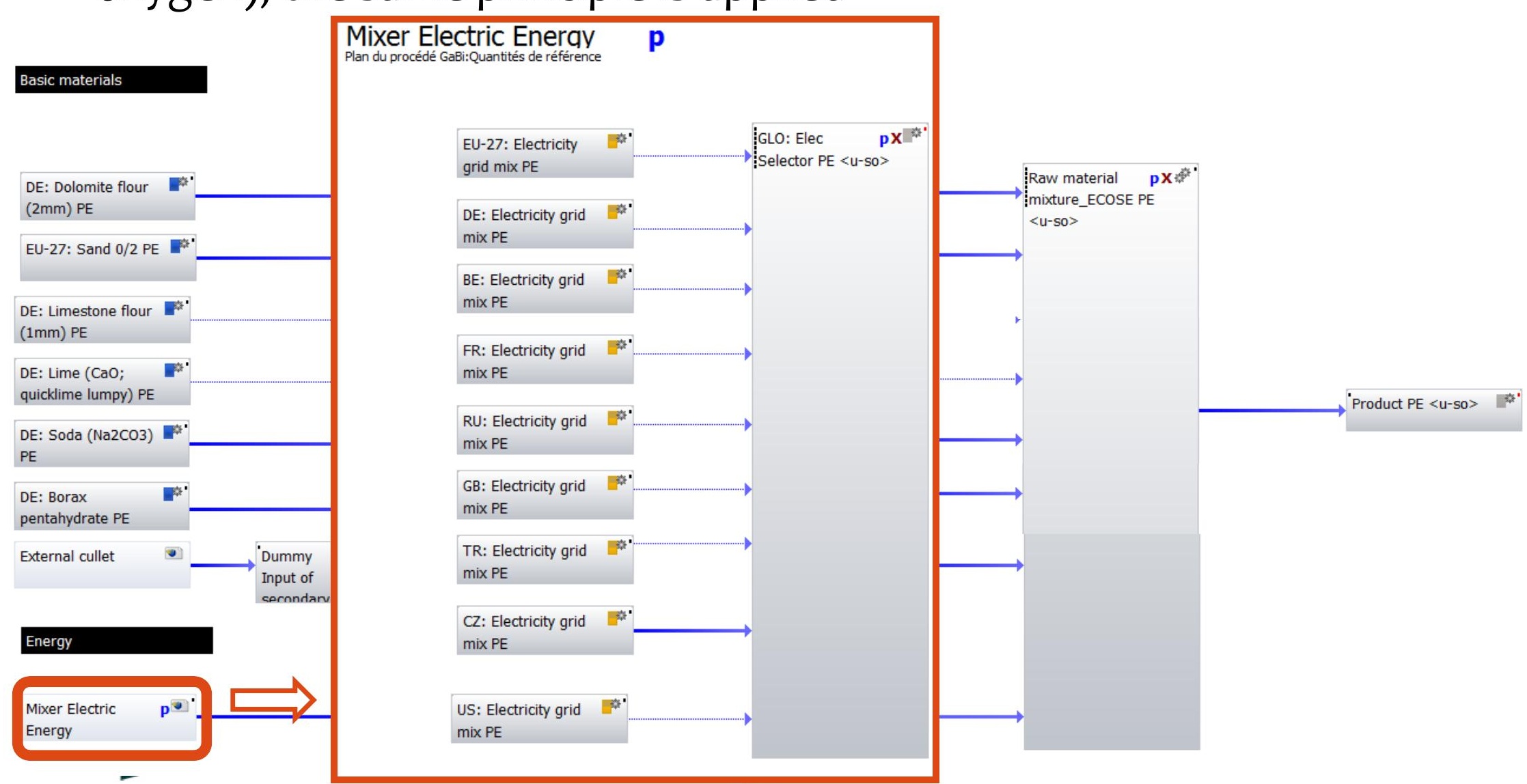




\section{Modeling}

- By reproducing the generic plan in a weighting plan:

Combination of factories possible

1. Plant weiahting: glass wool product - KNAUF INSULATION

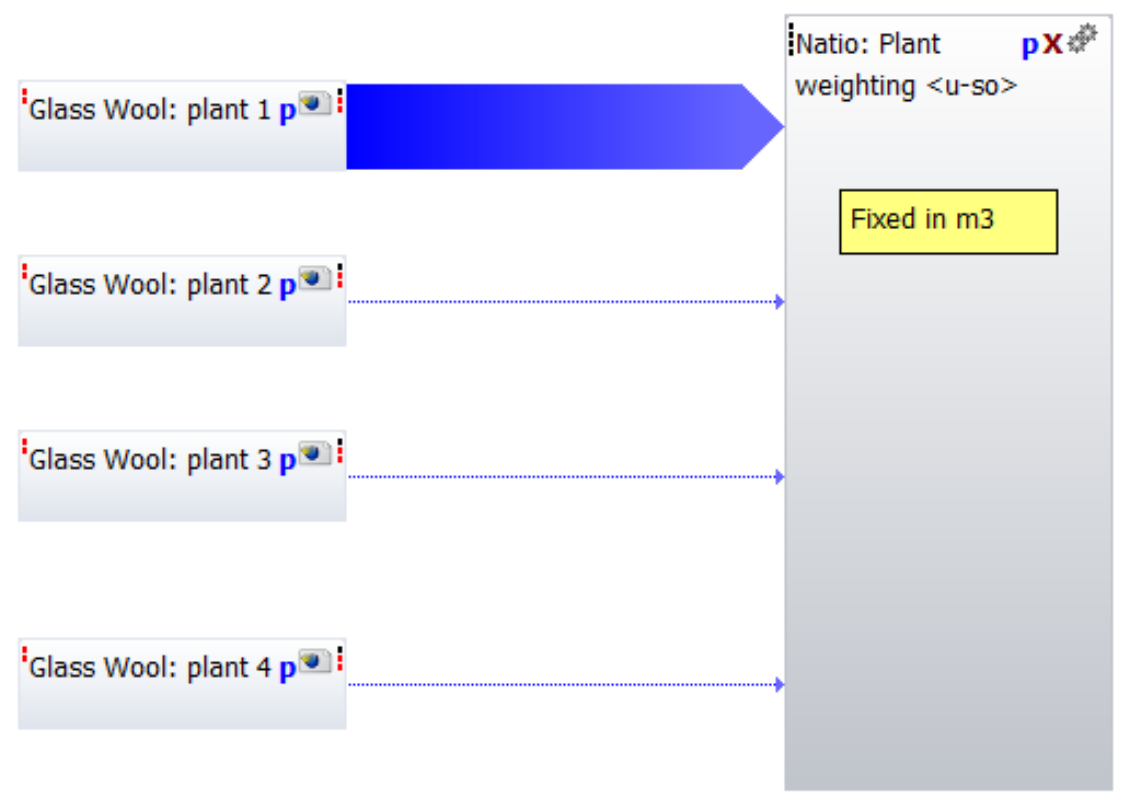




\section{Modeling}

- Parameters: Define

- Amount of each raw material

- Transport distance

- Energy consumption

- Origin of energy

- Amount of waste/co-product

$\rightarrow$ Plant parameters

- Example: the raw material mixture plan parameters 


\section{Modeling}

$\rightarrow$ Plant parameters

\section{- Example: the raw material mixture plan parameters}

\section{- Plant 1}

\begin{tabular}{l|l}
1 \\
\hline 1 \\
1 \\
1 \\
1 \\
1 \\
1 \\
1 \\
1 \\
1 \\
1 \\
1 \\
1 \\
1 \\
1 \\
1
\end{tabular}

IN

Out

\section{Raw material mixture}

$$
\text { Borate }
$$

Dolomite

Limestone

Calcinated limestone

Sand

Soda

External cullet

Electricity consumption at raw material m- Glass Wool: plant Elec_raw_mat

Raw material mixture

\section{Other}

Transport distance borate by SHIP

Transport distance Borate by TRUCK

Transport distance Dolomite
- Glass Wool: plant Borate

- Glass Wool: plant Dolomite

- Glass Wool: plant Limestone

- Glass Wool: plant Calcinated_lime

- Glass Wool: plant Sand

- Glass Wool: plant Soda

- Glass Wool: plant Ext_cullet

- Glass Wool: plant Raw_material_mi

- Glass Wool: plant Di_borate_ship

- Glass Wool: plant Di_Borate_truck

- Glass Wool: plant Di_Dolomite [kg] Borate consumption (raw material)

[kg] Dolomite consumption (raw material)

[kg] Limestone consumption (raw material)

[kg] Calcinated limestone consumption (raw material)

[kg] Sand consumption (raw material)

[kg] Soda consumption (raw material)

[kg] external cullet consumption (raw material)

[MJ] Electricity consumption for raw material

[kg] Raw materila mixture

[km] Transport distance by ship for borate (raw material) $[\mathrm{km}]$ Transport distance by truck for borate (raw material) $[\mathrm{km}]$ Transport distance for dolomite (raw material) 


\section{Modeling}

- Parameters: Define the product characteristics

- Dimension

- Type (blowing wool/ wool)

a Density

- Facing used

- Packaging used

- Production plant

$\rightarrow$ Product parameters 


\section{Modeling}

\section{$\rightarrow$ Product parameters}

\section{Product parameters \\ Product}

LOI

Gros calorific value

Product type

1. Plant weightincLOO

7,25

$\%$ of binder

- 1. Plant weightincGCV 1,35

Sous-ensemble Product type Glass wool

$[\mathrm{M}] / \mathrm{kg}]$ gross calorific value

ONLY RELEVANT for white wool product Sous-ensemble White wool appliciLoft

\section{Product dimension}

Density nominal

- 1. Plant weightinçdensity_nominal 24,8

$[\mathrm{Kg} / \mathrm{m} 3]$ of finished product to calculate grammage

Product length

- 1. Plant weightinçProduct_length 1,25

Product width

Thickness

- 1. Plant weightinçProduct_width 0,6

$\mathrm{Nb}$ Piece dallet

- 1. Plant weightinçThickness 80

- 1. Plant weightincNb PC Der dalle 120

$\mathrm{m}$

m

$\mathrm{mm}$ of finished product to calculate grammage oc Der pallet 


\section{Modeling}

$\rightarrow$ Product parameters

$\rightarrow$ Plant parameters

$\rightarrow$ Model allows to study every products from all production plants (or combination)! 


\section{Contents}

1. Introduction

2. The production process

3. LCA and modeling

4. Results and advantages

5. Conclusion 


\section{Advantages}

\section{- The data collection is simplified}

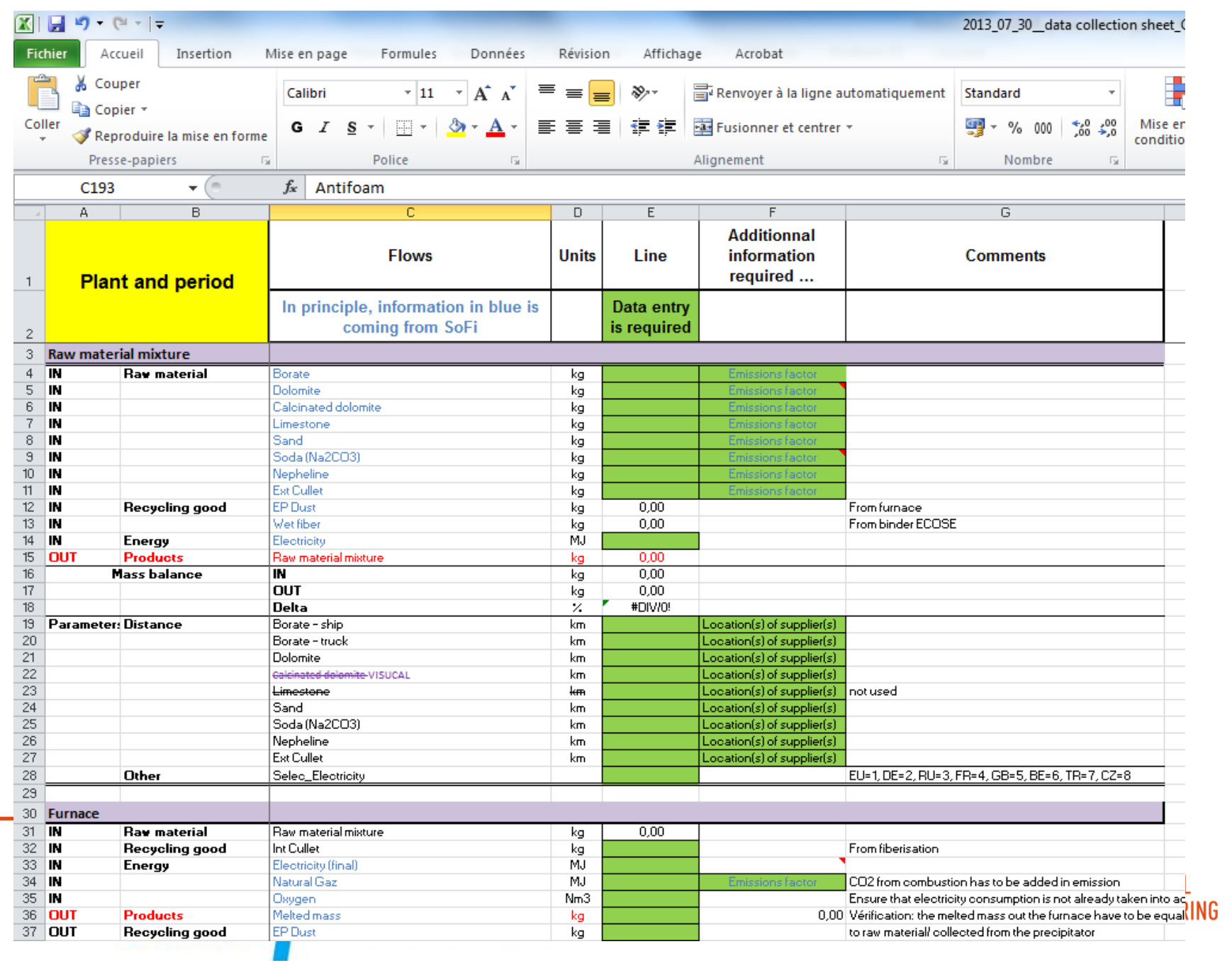




\section{Advantages}

\section{- The data collection is simplified}

\section{- EPD: faster}

\begin{tabular}{|c|c|c|c|c|}
\hline \multicolumn{5}{|l|}{ Product parameters } \\
\hline \multicolumn{5}{|l|}{ Product } \\
\hline LOI & \multicolumn{2}{|c|}{ - 1. Plant weightincLLOI } & 7,25 & $\%$ of binder \\
\hline Gros calorific value & \multicolumn{2}{|c|}{ 1. Plant weightinçGCV } & 1,35 & [M]/kg] gross calorific value \\
\hline Product type & Sous-ensemble & Product type & Glass wool & All the processes related to white wool in the plant \\
\hline ONLY RELEVANT for white wool product & Sous-ensemble & White wool applic & ciloft & \\
\hline \multicolumn{5}{|l|}{ Product dimension } \\
\hline Density nominal & \multicolumn{2}{|c|}{ 1. Plant weightinçdensity_nominal } & 24,8 & {$[\mathrm{Kg} / \mathrm{m} 3]$ of finished product to calculate grammage } \\
\hline Product length & \multicolumn{2}{|c|}{ 1. Plant weightinçProduct_length } & 1,25 & $\mathrm{~m}$ \\
\hline Product width & \multicolumn{2}{|c|}{ 1. Plant weightinçProduct_width } & 0,6 & $\mathrm{~m}$ \\
\hline Thickness & \multicolumn{2}{|c|}{ 1. Plant weightincThickness } & 80 & $\mathrm{~mm}$ of finished product to calculate grammage \\
\hline Nb Piece pallet & \multicolumn{3}{|c|}{ 1. Plant weiahtincNb PC der dalle 120} & DC Der Dallet \\
\hline
\end{tabular}


ENVIRONMENTAL PRODUCT DECLARATION

as per ISO 14025 and EN 15804

- EDP

\begin{tabular}{|c|c|}
\hline Owner of the Declaration & Knauf Insulation, d.0.0, Skofja Loka \\
\hline Programme holder & Institut Bauen und Umwelt e.V. (IBU) \\
\hline Publisher & Institut Bauen und Umwelt e.V. (IBU) \\
\hline Declaration number & EPD-KIN-20130163-CBC1-EN \\
\hline Issue date & 02.10 .2013 \\
\hline Valid to & 01.10 .2018 \\
\hline
\end{tabular}

DP-3 Multipurpose Rock Mineral Wool insulation

Knauf Insulation, d.o.o., Skofja Loka

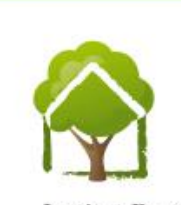

Institut Bauen

www.bau-umwelt.com / hitps:l/epd-online.com und Umwelt e.V.

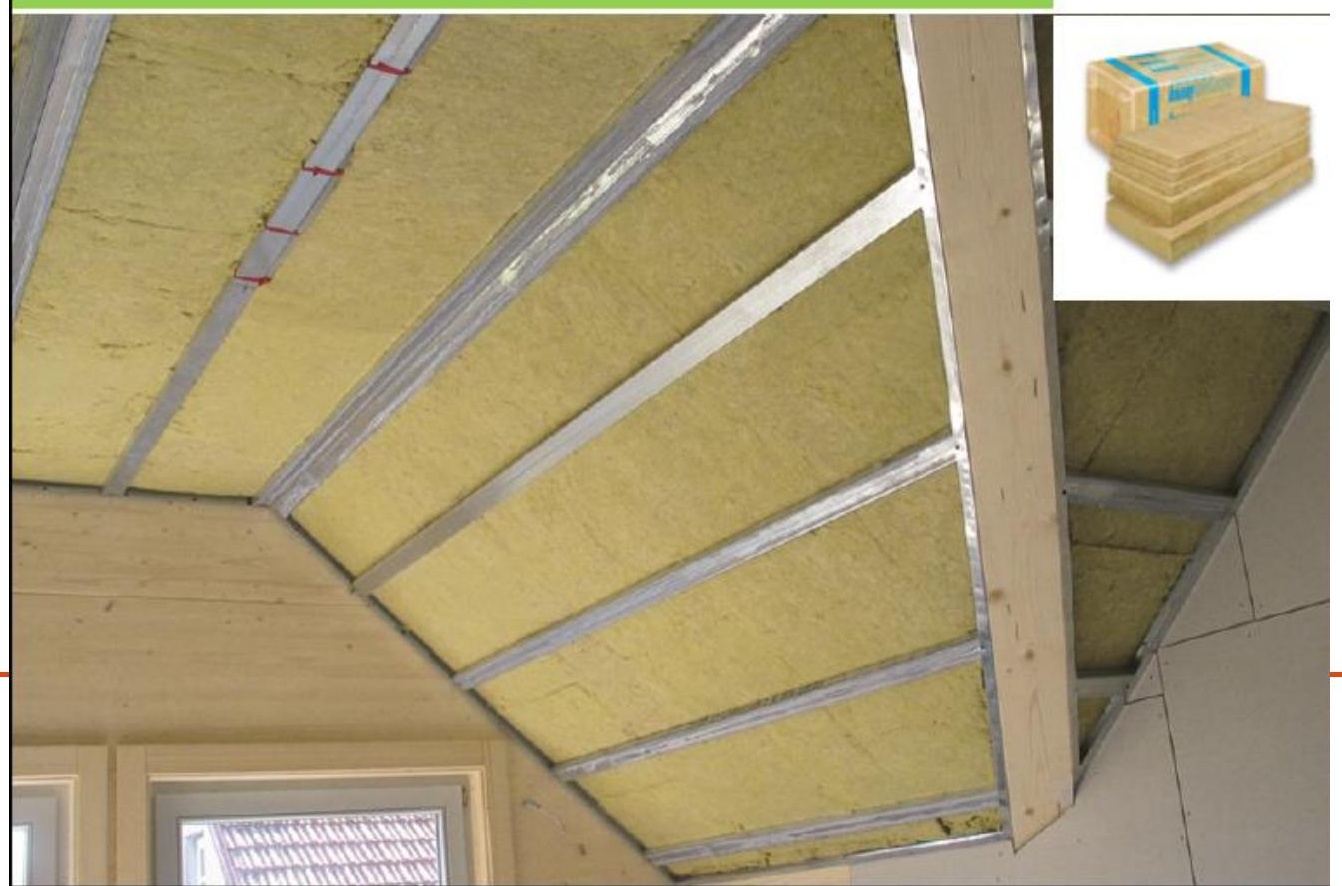




\section{Advantages}

- The data collection is simplified

- EPD: faster

- Eco-design 


\section{Advantages}

- Eco-design

a LCA : powerful multicriterea tool for eco-design

- Details

- Quantifies

- Avoid impact transfers (impact category / life cycle steps)

a Model: high flexibility and simplicity for the users

- LCA results: quick

- Several methods

- Identifies life cycle step high impact

- Comparison between different plants

- Alternative scenarios 


\section{Results}

- Example for a typical product for general construction applications with a density of $24.75 \mathrm{~kg} / \mathrm{m}^{3}$ (CML 2010)

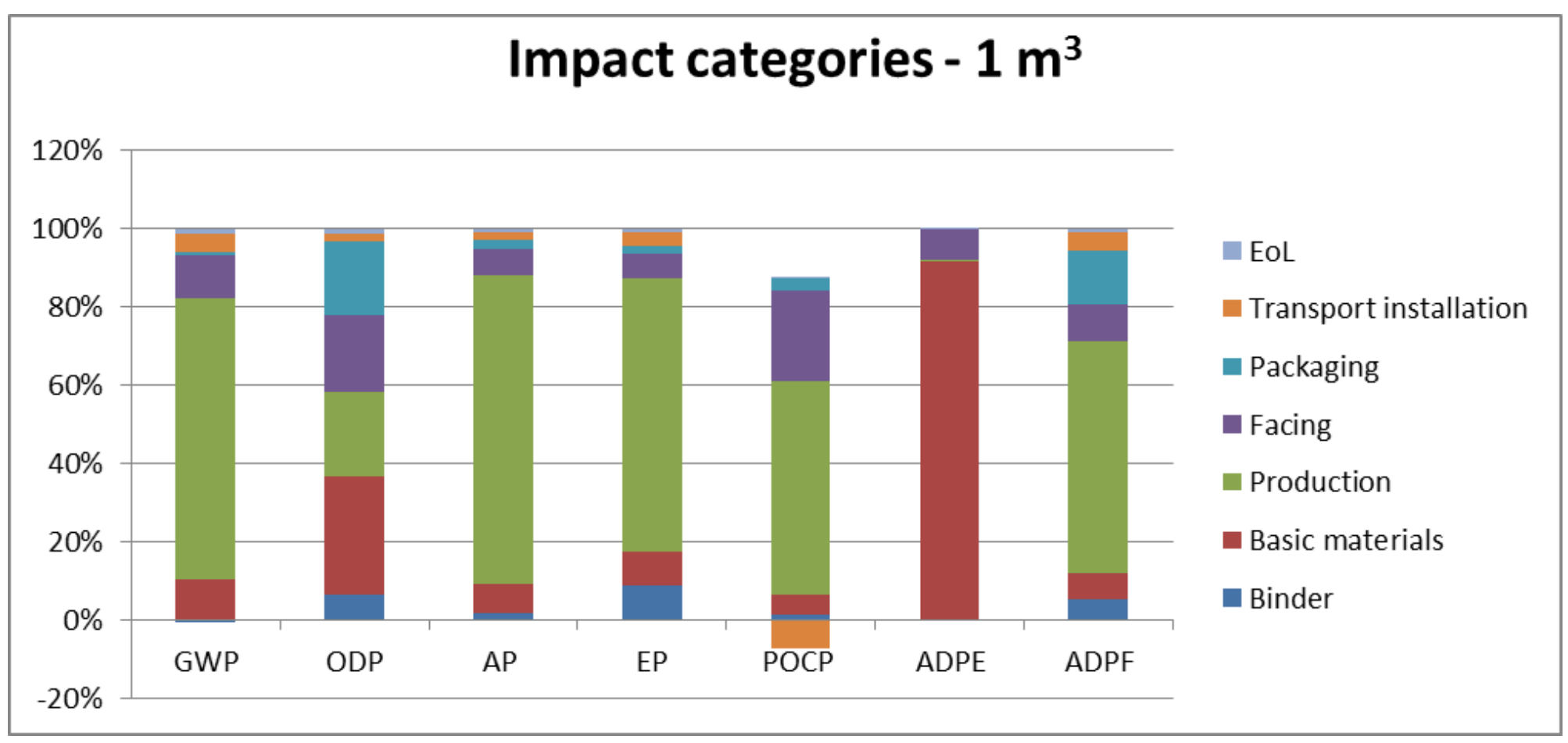




\section{Contents}

1. Introduction

2. The production process

3. LCA and modeling

4. Results and advantages

5. Conclusion 


\section{Conclusion}

- Development of a generic model

- Every glass mineral wool product

- All Knauf Insulation plant

- This model

a Simplifies the data collection procedure

a Allows

- EPD

- Eco-Design 


\section{Thank you for your attention!}

University of Liège LABORATORY of CHEMICAL ENGINEERING

Processes and Sustainable development saicha.gerbinet@ulg.ac.be 


\section{Modeling}

\section{- Example: used of thermal energy $\rightarrow$ fiberizing and forming}

Fiberizing and forming ECOSE Plan du procédé GaBi: Mass [kg]

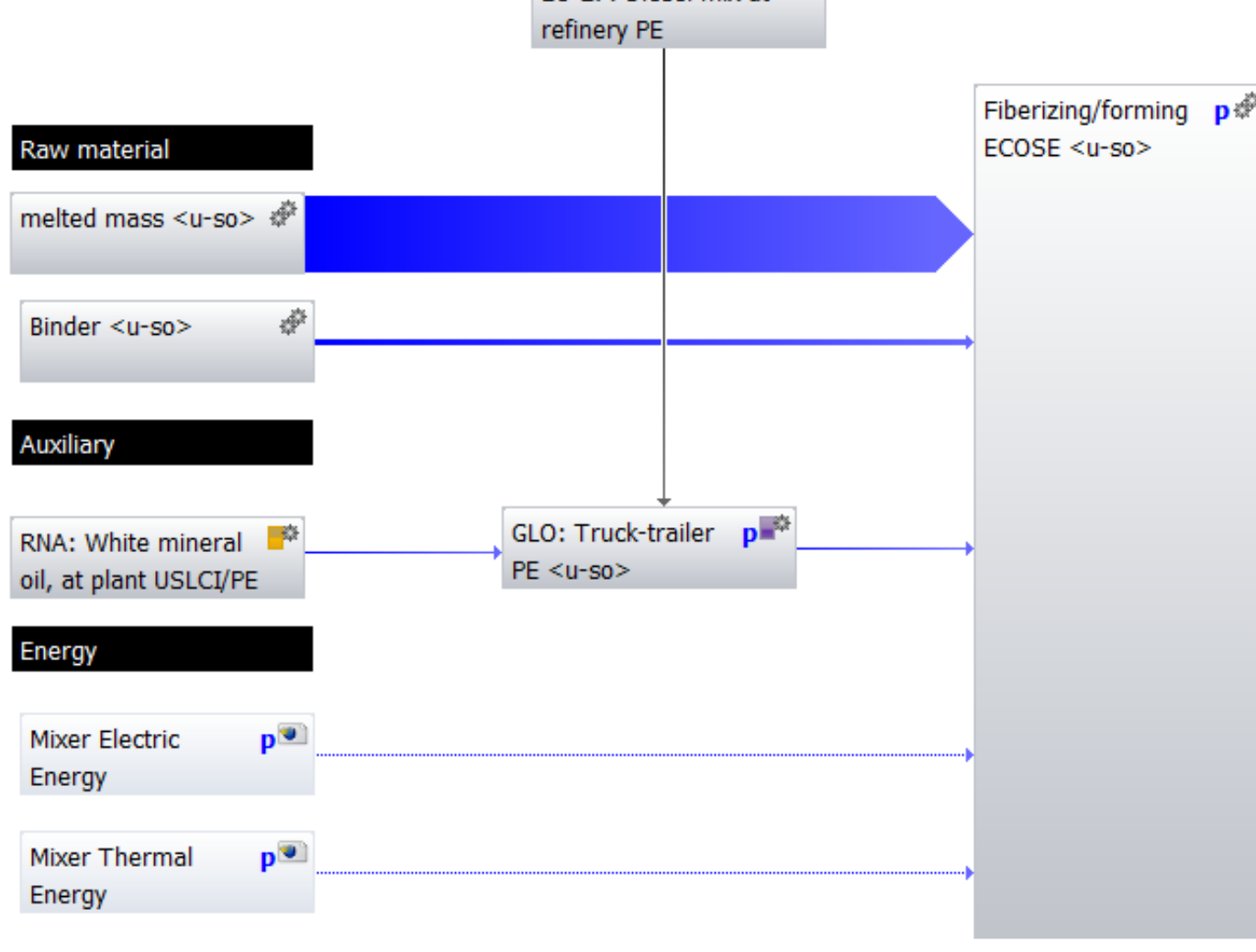

DE: Landfill (ECOSE

Binder) PE <p-agg>

GLO: Hazardous

waste (non-specific) (c

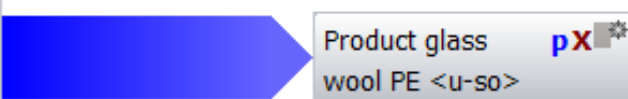

